\title{
O uso de metodologias ativas como facilitadoras do processo de desenvolvimento da empatia no acadêmico de Enfermagem - Uma abordagem Sociopoética
}

\author{
The Use of Active Methodologies as Facilitators of the Empathy Development Process in the Nursing \\ Academic - A Sociopoetic Approach \\ El uso de metodologías activas como facilitadores del proceso de desarrollo de la empatía en el ámbito \\ académico de enfermería: un enfoque sociopoético
}

Helga Rocha Pitta Portella Figueiredo ${ }^{1 *}$, Claudia Mara de Mello Tavares²

Como citar esse artigo. Figueiredo, HRPP; Tavares, CMM. O uso de metodologias ativas como facilitadoras do processo de desenvolvimento da empatia no acadêmico de enfermagem - Uma abordagem Sociopoética. Revista Pró-UniverSUS. 2019 Jul./Dez.; 10 (2): 131-133.

\begin{abstract}
Resumo
Objetivos: Propor estratégias de ensino com base nas metodologias ativas e na sociopoética para o desenvolvimento da empatia no acadêmico de enfermagem; Descrever como se dá o preparo do acadêmico de enfermagem para desenvolver a competência empática junto aos pacientes a partir das metodologias ativas; Experimentar dinâmicas de ensino da empatia junto aos acadêmicos de enfermagem; Avaliar a contribuição das dinâmicas utilizadas para a formação do comportamento empático junto aos acadêmicos de enfermagem e propor uma abordagem metodológica que colabore com o aprimoramento do ensino da aprendizagem de competências empáticas na formação do enfermeiro.Método: Estudo qualitativo, descritivo, com abordagem sociopoética. A escolha por esta abordagem nos permite uma produção coletiva de conceitos e problemas que mobilizam o grupo de sujeitos envolvidos na pesquisa.Resultados esperados: Acredita-se que esse estudo contribua com a formação de enfermeiros para atuarem futuramente com pensamento crítico-reflexivo e empático para compreensão e resolutividade dos problemas dos usuários do SUS. Produto:Esta pesquisa almeja propor uma estratégia de abordagem metodológica que colabore com o aprimoramento do ensino da aprendizagem de competências empáticas na formação do enfermeiro.

Palavras-chave: Empatia, Educação em enfermagem, Aprendizagem baseada em problemas.
\end{abstract}

\begin{abstract}
Objectives: To propose teaching strategies based on active methodologies and sociopoetics for the development of empathy in nursing students; Describe how the nursing academic prepares to develop empathic competence with patients based on active methodologies; Experience the dynamics of teaching empathy with nursing students; To evaluate the contribution of the dynamics used for the formation of empathic behavior among nursing students and to propose a methodological approach that collaborates with the improvement of the teaching of the learning of empathic skills in the formation of nurses. Method: Qualitative, descriptive study with sociopoetic approach. The choice for this approach allows us a collective production of concepts and problems that mobilize the group of subjects involved in the research. Expected Results: It is believed that this study contributes to the training of nurses to act in the future with critical-reflective and empathic thinking to understand and solve the problems of SUS users. Product: This research aims to propose a methodological approach strategy that collaborates with the improvement of the teaching of the learning of empathic skills in the formation of nurses.
\end{abstract}

Keywords: Empathy, Nursing education, Problem-based learning.

\section{Resumen}

Objetivos: Proponer estrategias de enseñanza basadas en metodologías activas y sociopoéticas para el desarrollo de la empatía en académicos de enfermería;Describir cómo el académico de enfermería debe se preparar para desarrollar la habilidad empática con los pacientes basadas en las metodologías activas;Experimentar la dinámica de enseñanza de empatía en académicos de enfermería;Evaluar la contribución de la dinámica utilizada para la formación del comportamiento empático entre académicos de enfermería y proponer un acercamiento metodológico que contribuya con la mejora de la enseñanza de habilidades empáticas en la formación de los enfermeros.Método:Estudio cualitativo descriptivo con acercamiento sociopoético.La elección de este enfoque nos permite una producción colectiva de conceptos y problemas que movilizan lo grupo de sujetos involucrados en la investigación. Resultado esperado:Se cree que este estudio contribuye a la formación de enfermeros para actuar en el futuro con pensamiento crítico-reflexivo y empático para comprensión y resolución de los problemas de los usuarios del SUS. Producto:Esta investigación tiene como objetivo proponer una estrategia de acercamiento metodológico que contribuya con a la mejora de la enseñanza de habilidades empáticas en la educación de la enfermería.

Palabras clave: Empatía, Educación en enfermería, Aprendizaje basado en problemas.

Afiliação dos autores: 1. Enfermeira, Mestranda no Programa Profissional Ensino na Saúde, Universidade Federal Fluminense, Niterói, Rio de Janeiro, Brasil. ORCID: https://orcid. org/0000-0003-2899-1988

2. Enfermeira, Professora Titular da Escola de Enfermagem -UFF. Doutora em Enfermagem. Brasil. ORCID: https://orcid.org/0000-0002-8416-6272

* E-mail de correspondência: helgapitta@gmail.com 


\section{Introdução}

No Brasil dos últimos anos houve um consistente esforço para reorganizar e incentivar a atenção básica, como estratégia de substituição do modelo tradicional de organização do cuidado em saúde, historicamente centrado na doença e no atendimento hospitalar.

Em 1994, foi implantado o Programa Saúde da Família (PSF), mais tardiamente renomeado como Estratégia de Saúde da Família (ESF), que visava o estabelecimento de uma atenção básica resolutiva e de qualidade, reafirmando os princípios constitucionais estabelecidos para o Sistema Único de Saúde (SUS), na medida em que se busca, por meio desta atenção, reforçar a universalidade do acesso, da equidade e da integralidade das ações ${ }^{1}$.

Posteriormente em 2003, a Política Nacional de Humanização (PNH) busca trazer mudanças nos modos de cuidar, trazendo o conceito de clínica ampliada, uma ferramenta teórica e prática que busca contribuir para uma abordagem clínica do adoecimento e do sofrimento, valorizando a singularidade do sujeito e a complexidade do processo saúde/doença, utilizando recursos que permitam enriquecimento dos diagnósticos, entre eles a percepção dos afetos produzidos na relação entre profissional de saúde e paciente ${ }^{2}$.

Reconhecendo a necessidade da formação de profissionais preocupados com o cuidado humanizado, atualmente, discussões apontam para a utilização de novas práticas pedagógicas e as instituições de ensino superior têm sido estimuladas a reconstruir seu papel social e valorizar a qualidade da assistência no trabalho em saúde, adotando tais inovações ${ }^{3}$.

Diante de toda esta movimentação em busca da formação de futuros enfermeiros comprometidos com a humanização da assistência ainda destacamos o pensamento de autores como Takaki e Sant'ana (2011), que afirmam que a enfermagemé conceituada como a arte de cuidar, e neste sentido, a empatia habilidade essencial do cuidar, constitui-se um componente fundamental do tratamento dispensado por este profissional ao cliente ${ }^{4}$.

\section{Objetivos}

- Propor estratégias de ensino com base nas metodologias ativas e na sociopoética para o desenvolvimento da empatia no acadêmico de enfermagem;

- Descrever como se dá o preparo do acadêmico de enfermagem para desenvolver a competência empática junto aos pacientes a partir das metodologias ativas;

- Experimentar dinâmicas de ensino da empatia junto aos acadêmicos de enfermagem;

- Propor uma abordagem metodológica que colabore com o aprimoramento do ensino de competências empáticas na formação do enfermeiro.
Método

Trata-se de estudo qualitativo, descritivo, com abordagem sociopoética. A escolha pela pesquisa qualitativa decorre do desejo de investigara subjetividade de cada um dos sujeitos envolvidos na pesquisa.

Para Minayo (2001), a pesquisa qualitativa trabalha com o universo de significados, motivos, aspirações, crenças, valores e atitudes, o que corresponde a um espaço mais profundo das relações, dos processos e dos fenômenos que não podem ser reduzidos à operacionalização de variáveis ${ }^{5}$.

Já a Sociopoética como uma prática filosófica tem por objetivo a produção de conceitos sobre os temas e problemas que mobilizam os grupos com os quais trabalha.

Assim, em grupo, utilizando, em suas pesquisas, técnicas artísticas e o corpo todo para produzir conceitos, a Sociopoética também acredita que usar apenas a razão na pesquisa é reduzir a capacidade de criação, de invenção no ato de conhecer. Com relação ao seu corpo teórico, a sociopoética fundamenta-se nos seguintes princípios: o grupo pesquisador como dispositivo; a importância do corpo como fonte de conhecimento; o papel da criatividade de tipo artística no aprender, no conhecer e no pesquisar; a ênfase no sentido ético no processo de construção dos saberes ${ }^{6}$.

Os participantes do estudo serão estudantes do curso de graduação em enfermagem. Os critérios para inclusão dos participantes serão: discentes do $7^{\circ}$ e $8^{\circ}$, (pois já cursaram a maior parte das disciplinas teóricas e práticas da graduação, portanto acredita-se que estes tenham uma maior experimentação na utilização das metodologias ativas ofertadas. Outro critério será a escolha por alunos regularmente matriculados no curso, e que também aceitem participar da pesquisa assinando o termo de consentimento livre e esclarecido.

Como critério de exclusão estabeleceu-se que os alunos dos mesmos períodos que não concordarem em praticar ou que porventura estiverem matriculados em outro período da graduação que não os mencionados. Não se optou por convidar para a pesquisa graduandos dos dois últimos semestres da graduação $\left(9^{\circ}\right.$ e $\left.10^{\circ}\right)$ pois estes por já estarem em campos de estágio distintos, atuado todos os dias da semana, teríamos dificuldades relacionadas à escolha e marcação das datas e horários de agendamento das etapas de produção dos dados.

Serão então organizados três grupos onde para cada, será ofertada uma estratégia metodológica diferenciada com intuito de observar se alguma destas se apresenta como facilitadora no desenvolvimento do domínio da empatia nestes discentes.

Antes desta etapa, vale ressaltar que a pesquisa será submetida à apreciação do Comitê de Ética em Pesquisa da Universidade Federal Fluminense - UFF e 
da IES coparticipante da pesquisa.

A posteriori, o tratamento e a análise dos dados coletados durante as oficinas realizadas com os grupos, serão trabalhados e categorizados de acordo com a temática.

Para atender a necessidade da pesquisa, utilizaremos como campo de estudo uma instituição de ensino superior (IES) particular, do município do Rio de Janeiro. A escolha deste local vai de encontro a oportunidade de estar inserida no quadro de docentes ativos desta IES e no desejo de contribuir na criação de estratégias que possam serimplementadas para a melhoria da educação e a posteriori da assistência, no que tange aos aspectos emocionais e especificamente empáticos na formação do enfermeiro, nesta instituição.

A proposta de pesquisa será apresentada ao Programa de Pós-graduação do Mestrado Profissional em Ensino na Saúde Formação Docente Interdisciplinar para o SUS, da Escola de Enfermagem Aurora de Afonso Costa (EEAAC)- Universidade Federal Fluminense (UFF) Campus Niterói, linha de Pesquisa: Formação pedagógica em Saúde, esse projeto de pesquisa será submetido ao Comitê de Ética e Pesquisa da Universidade Federal Fluminense.

\section{Resultados esperados}

Acredita-se que esse estudo contribua com a formação de enfermeiros para atuarem na Atenção Primária à Saúde, com pensamento crítico-reflexivo e empático para compreensão e resolutividade dos problemas dos usuários do SUS. Como produto dessa pesquisa almeja-se propor um estratégia de abordagem metodológica que colabora com o aprimoramento do ensino da aprendizagem de competências empáticas na formação do enfermeiro.

\section{Referências}

1. Brasil. Ministério Da Saúde. Portaria No 2.436, De 21 De Setembro De 2017. Aprova A Política Nacional De Atenção Básica, Estabelecendo A Revisão De Diretrizes Para A Organização Da Atenção Básica, No Âmbito Do Sistema Único De Saúde (Sus). Brasília, Df: Ministério Da Saúde, 2017

2. Brasil. Ministério Da Saúde. Secretaria De Atenção À Saúde. Núcleo Técnico Da Política Nacional De Humanização. Humanizasus: Documento Base Para Gestores E Trabalhadores Do Sus. 4.Ed. Brasília: Editora Do Ministério Da Saúde, 2007.

3. Sobral, Fernanda Ribeiro; Campos, Claudinei José Gomes. Utilização De Metodologia Ativa No Ensino E Assistência De Enfermagem Na Produção Nacional: Revisão Integrativa. Rev. Esc. Enferm. Usp, São Paulo, V. 46, N. 1, P. 208-218, Feb. 2012

4. Takaki, Maria Harue; Sant'ana, Débora De Mello Gonçalves. A Empatia Como Essência No Cuidado Prestado Ao Cliente Pela Equipe De Enfermagem De Uma Unidade Básica De Saúde. Cogitare Enfermagem, [S.L.], V. 9, N. 1, Jun. 2004.

5. MINAYO, M. C. S. O desafio do conhecimento: pesquisa qualitativa em saúde. 8.ed. São Paulo: Hucitec, 2004.
6. Gauthier, Jacques. Sociopoética-Encontro Entre Arte, Ciência E Democracia Na Pesquisa Em Ciências Humanas E Sociais, Enfermagem E Educação. Rio De Janeiro: Escola Anna Nery/Ufrj, 1999. 